\title{
Synthesis of robust control algorithms for linear interval dynamic systems in the electric power industry
}

\author{
Igor Boldyrev*, Dmitry Tonn, Sergey Goremykin, Nikolay Sitnikov, Alexander Mukonin, and Viktor Trubetskoy \\ Voronezh State Technical University, 20-letiya Oktyabrya str., 84, 394006, Voronezh, Russia
}

\begin{abstract}
When studying the objects of management in the field of electric power, we meet with various inaccuracies in determining their parameters. One of the methods of dealing with uncertainties is the use of various estimates of the parameters of the control object. modern science has developed various methods for assessing the uncertain parameters of the control object in the electric power industry. parameter uncertainty occurs when the set of parameters of the control object is more than one point. If this set is defined using probabilistic characteristics, then this is the so-called probabilistic uncertainty of the object parameters. If the boundaries of the intervals in which they are enclosed are known for the object parameters, then such uncertainty is called interval uncertainty. if the object parameters are set using the membership function, then the theory of fuzzy logic is used. Interval determination of parameters of the control object is used when working with values for which only the boundaries of the intervals within which their values are enclosed are known. The interval approach in the description of object parameters is used to account for rounding and errors that occur during calculations on a computer and is a strong method in the representation of objects with uncertain parameters, which are very common in the electric power industry. The reason why interval systems are used is the incompleteness of information about the control object, errors in measuring the parameters of the object, linearization errors, and so on. Various problems of the classical theory of automatic control allow us to replace the concentrated parameters with their interval analogues. Many interval problems are adequate for practical applications. The synthesis of linear quadratic regulators refers to the classical method, which allows us to obtain regulators that minimize the integral quality criterion with respect to the resource of regulated and regulatory quantities. In this paper, we study the possibility of synthesizing linear quadratic regulators for electric power facilities specified in intervals. This application of two well-known methods makes it possible to work with nonlinear objects using the classical linear control theory.
\end{abstract}

\section{Introduction}

In the continuous process of generation, distribution, transmission and consumption of electric energy in the electric power system, there is a change in various types of loads, switching of circuit elements, changes in the production of active and reactive power, short circuits and transients, unauthorized disconnections of electrical equipment, operation of relay protection and emergency automation, so the system is exposed to small and large impacts. Power system responds to external stimuli by changing settings, such as, the initial phase and the value of the modules of voltage, flow capacity, changes in currents, speed of rotation of synchronous and asynchronous machines, etc. the Amount and composition of the responses of the power system depends both on the magnitude and type of external disturbances, and from properties of the system, the structure schema, values and types of parameters. Like any complex system, the electric power system is extremely heterogeneous, so the parameters of the operating mode of some of its elements react more strongly to impacts.

An important criterion necessary for reliable, highquality and economical management of electric power systems is a valid mathematical representation of the sensitivity of the parameters of its operating mode to external influences. in connection with all the above, it can be concluded that the objects of electric power systems are represented in the form of linear differential equations with interval parameters [1-5].

In this paper, we consider a method for evaluating the integral control quality criterion for linear interval dynamical systems covered by feedback, which is a class of linear quadratic regulators. All of the above, within the framework of this technique, allows us to talk about the synthesis of robust control algorithms for linear interval dynamic systems in the electric power industry.

\footnotetext{
* Corresponding author: boldyrev igor@mail.ru
} 


\section{Materials and methods}

\subsection{Formulation of the problem}

Equation (1) represents a linear model of the state space

$$
\dot{x}=(F+\Delta F) x+B u
$$

where $\mathrm{x} \in R^{n}$ state vector, $\mathrm{u} \in R^{m}$ control signal and $F=\left(f_{i j}\right) \in R^{n \times n}, \Delta F=\left(\Delta f_{i j}\right) \in R^{n \times n}$ and $B=\left(b_{i}\right) \in R^{n}$

matrices of corresponding dimensions.

Let the matrix elements $\Delta F$ be represented as

$$
\forall(i, j) \in \overline{\{1, n\}} \Delta f_{i j}=\sum_{m=1}^{p} \mu_{i j}^{m} \cdot \delta_{m}
$$

Where $\delta_{m}$, is an unknown constant and such that

$$
\forall m \in \overline{\{1, p\}} \delta_{m}^{\min } \leq \delta_{m} \leq \delta_{m}^{\max }
$$

Where $\mu_{i j}^{m}, \delta_{m}^{\min }, \delta_{m}^{\max }-$ known constants

$$
Q-K B R^{-1} B^{T} K^{T}+K F+F^{T} K^{T}=0
$$

Equation (4) is known as the Time Continuous Riccati Algebra Equation.

If $\Delta F \equiv[0]$ then optimal control can be represented as

$$
u=-R^{-1} B^{T} K^{T} x
$$

This is an optimal controller problem defined as a problem based on optimal control $u$ on the interval $[0,+$ $\infty]$ such that the next cost function is minimized.

$$
J=\int_{0}^{\infty}\left(x^{T} Q x+u^{T} R u\right) d t=x_{0}^{T} K x_{0}
$$

Where $Q \in R^{n \times n}$ symmetric non-negative definite matrix and $K \in R^{n \times n} R \in R^{m \times m} \quad$ symmetric positive definite matrices. We also note that $x(0)=x_{0}$ initial conditions of the state space $x(t) \in R^{n}$.

Suppose that the matrix $\Delta F \neq[0]$ then equation (1) is called linear interval dynamical system. However, if the matrix $\Delta F \neq[0]$ then equation u (5) does not minimize the cost function (6).

In this case, it is necessary to obtain an estimate of the value of the cost function (6).

\subsection{Cost function for LIDS with variable structure}

Let the matrix $\Delta F$ be nonzero and $\Omega$ will be a set of matrices $A^{k}=\left(a_{i j}^{k}\right) \in R^{n \times n}$ such that

$$
\forall k \in \overline{\left\{0,2^{p}-1\right\}}: a_{i j}^{k}=\sum_{m=1}^{p} \mu_{i j}^{m} \cdot \delta_{m}^{k}+f_{i j}
$$

Let

$$
a_{i j}^{0}=\sum_{m=1}^{p} \mu_{i j}^{m} \cdot \delta_{m}^{\min }+f_{i j}
$$

Note that the set $\Omega$ comprises of $2^{p}$ elements.

Lemma 1. Assume that

$$
A=F+\Delta F \text { or } a_{i j}=f_{i j}+\Delta f_{i j}
$$

If $A=\left(a_{i j}\right)$ is a matrix that satisfies the conditions (2), (3) then

$$
\begin{gathered}
A=\sum_{k=0}^{2^{p}-1} \lambda_{k} \cdot A^{k} \text { or } a_{i j}=\sum_{k=0}^{2^{p}-1} \lambda_{k} \cdot a_{i j}^{k} \\
\lambda_{k} \geq 0\left(k=\overline{0 ; 2^{p}-1}\right) \\
\sum_{k=0}^{2^{p}-1} \lambda_{k}=1
\end{gathered}
$$

\section{Proof}

Without loss of generality, we write

$$
\frac{\tilde{\delta}_{i}}{\bar{\delta}_{i}} \geq \frac{\tilde{\delta}_{i+1}}{\bar{\delta}_{i+1}}, \forall i \in \overline{\{1, p-1\}}
$$

where $\tilde{\delta}_{k}=\delta_{k}-\delta_{k}^{\min } ; \bar{\delta}_{k}=\delta_{k}^{\max }-\delta_{k}^{\min }$,

if

$$
\begin{aligned}
& \lambda_{0}=\left(1-\frac{\tilde{\delta}_{1}}{\bar{\delta}_{1}}\right) ; \lambda_{k}=\left(\frac{\tilde{\delta}_{k}}{\bar{\delta}_{k}}-\frac{\tilde{\delta}_{k+1}}{\bar{\delta}_{k+1}}\right), \forall k \in \overline{\{1, p-1\}} \\
& \lambda_{p}=\frac{\tilde{\delta}_{p}}{\bar{\delta}_{p}} ; \lambda_{m}=0, \forall m \in \overline{\left\{p+1,2^{p}-1\right\}}
\end{aligned}
$$

Then the conditions (10), (11), (12) can be performed. We will show it below.

From (14) it follows that $\lambda_{k} \geq 0\left(k=\overline{0 ; 2^{p}-1}\right)$.

If

$\sum_{k=0}^{2^{p}-1} \lambda_{k} \stackrel{(13)}{=}\left(1-\frac{\tilde{\delta}_{1}}{\bar{\delta}_{1}}\right)+\sum_{k=1}^{p-1}\left(\frac{\tilde{\delta}_{k}}{\bar{\delta}_{k}}-\frac{\tilde{\delta}_{k+1}}{\bar{\delta}_{k+1}}\right)+\frac{\tilde{\delta}_{p}}{\bar{\delta}_{p}}=\left(1-\frac{\tilde{\delta}_{1}}{\bar{\delta}_{1}}\right)+\left(\frac{\tilde{\delta}_{1}}{\bar{\delta}_{1}}-\frac{\tilde{\delta}_{p}}{\bar{\delta}_{p}}\right)+\frac{\tilde{\delta}_{p}}{\bar{\delta}_{p}}=1$

then this confirms that $\sum_{k=0}^{2^{p}-1} \lambda_{k}=1$.

Let

$$
\forall(i, j) \in \overline{\{1, n\}}, \quad a_{i j}^{k}=\sum_{m=1}^{p} \mu_{i j}^{m} \cdot \delta_{m}^{k}+f_{i j}
$$

where $\delta_{m}^{k}=\left\{\begin{array}{lll}\delta_{m}^{\max } & \text { if } & m \leq k \\ \delta_{m}^{\min } & \text { if } & m>k\end{array}\right.$;

$$
\tilde{\delta}_{m}^{k}=\left\{\begin{array}{ccc}
\delta_{m}^{\max }-\delta_{m}^{\min } & \text { if } & m \leq k \\
0 & \text { if } & m>k
\end{array},\right.
$$


Such that from (15), (16) we see that

$$
\begin{aligned}
& \sum_{k=0}^{2^{p}-1} \lambda_{k} \cdot a_{i j}^{k} \stackrel{(14)}{=}\left(1-\frac{\tilde{\delta}_{1}}{\bar{\delta}_{1}}\right) a_{i j}^{0}+\sum_{k=1}^{p-1}\left(\frac{\widetilde{\delta}_{k}}{\bar{\delta}_{k}}-\frac{\tilde{\delta}_{k+1}}{\bar{\delta}_{k+1}}\right) a_{i j}^{k}+\frac{\tilde{\delta}_{p}}{\bar{\delta}_{p}} a_{i j}^{p}= \\
& \stackrel{(15)}{=}\left(1-\frac{\hat{\delta}_{1}}{\bar{\delta}_{1}}\right) a_{i j}^{0}+\sum_{k=1}^{p-1}\left(\left(\frac{\hat{\delta}_{k}}{\bar{\delta}_{k}}-\frac{\hat{\delta}_{k+1}}{\bar{\delta}_{k+1}}\right)\left(a_{i j}^{0}+\sum_{m=1}^{p} \mu_{i j}^{m} \cdot \hat{\delta}_{m}^{k}\right)\right)+\frac{\delta_{p}}{\bar{\delta}_{p}}\left(a_{i j}^{0}+\sum_{m=1}^{p} \mu_{i j}^{m} \cdot \hat{\delta}_{m}^{p}\right)= \\
& =\sum_{k=0}^{p} \lambda_{k} a_{i j}^{0}+\sum_{k=1}^{p-1}\left(\left(\frac{\tilde{\delta}_{k}}{\bar{\delta}_{k}}-\frac{\tilde{\delta}_{k+1}}{\bar{\delta}_{k+1}}\right)\left(\sum_{m=1}^{p} \mu_{i j}^{m} \cdot \tilde{\delta}_{m}^{k}\right)\right)+\frac{\tilde{\delta}_{p}}{\bar{\delta}_{p}}\left(\sum_{m=1}^{p} \mu_{i j}^{m} \cdot \tilde{\delta}_{m}^{p}\right)^{(12)}= \\
& \stackrel{(12)}{=} a_{i j}^{0}+\sum_{k=1}^{p-1}\left(\left(\frac{\tilde{\delta}_{k}}{\bar{\delta}_{k}}-\frac{\tilde{\delta}_{k+1}}{\bar{\delta}_{k+1}}\right)\left(\sum_{m=1}^{p} \mu_{i j}^{m} \cdot \widetilde{\delta}_{m}^{k}\right)\right)+\frac{\widetilde{\delta}_{p}}{\bar{\delta}_{p}}\left(\sum_{m=1}^{p} \mu_{i j}^{m} \cdot \widetilde{\delta}_{m}^{p}\right) \stackrel{(16)}{=} \\
& \stackrel{(16)}{=} a_{i j}^{0}+\sum_{k=1}^{p-1}\left(\left(\frac{\tilde{\delta}_{k}}{\bar{\delta}_{k}}-\frac{\tilde{\delta}_{k+1}}{\bar{\delta}_{k+1}}\right)\left(\sum_{m=1}^{k} \mu_{i j}^{m} \cdot \bar{\delta}_{m}\right)\right)+\frac{\tilde{\delta}_{p}}{\bar{\delta}_{p}}\left(\sum_{m=1}^{p} \mu_{i j}^{m} \cdot \bar{\delta}_{m}\right)= \\
& =a_{i j}^{0}+\sum_{k=1}^{p-1}\left(\left(\bar{\delta}_{k}\right)\left(\sum_{m=1}^{k} \mu_{i j}^{m} \cdot \bar{\delta}_{m}\right)\right)-\sum_{k=1}^{p-1}\left(\left(\bar{\delta}_{k+1}\right)\left(\sum_{m=1}^{k} \mu_{i j}^{m} \cdot \bar{\delta}_{m}\right)\right)+\frac{\hat{\delta}_{p}}{\bar{\delta}_{p}}\left(\sum_{m=1}^{p} \mu_{i j}^{m} \cdot \bar{\delta}_{m}\right)= \\
& =a_{i j}^{0}+\sum_{k=1}^{p-1}\left(\left(\bar{\delta}_{k}\right)\left(\sum_{m=1}^{k} \mu_{i j}^{m} \cdot \bar{\delta}_{m}\right)\right)-\sum_{k=2}^{p}\left(\left(\frac{\tilde{\delta}_{k}}{\bar{\delta}_{k}}\right)\left(\sum_{m=1}^{k-1} \mu_{i j}^{m} \cdot \bar{\delta}_{m}\right)\right)+\frac{\hat{\delta}_{p}}{\bar{\delta}_{p}}\left(\sum_{m=1}^{p} \mu_{i j}^{m} \cdot \bar{\delta}_{m}\right)= \\
& =a_{i j}^{0}+\sum_{k=2}^{p-1}\left(\left(\frac{\bar{\delta}_{k}}{\bar{\delta}_{k}}\right)\left(\sum_{m=1}^{k-1} \mu_{i j}^{m} \cdot \bar{\delta}_{m}\right)\right)+\left(\frac{\bar{\delta}_{1}}{\bar{\delta}_{1}}\right)\left(\mu_{i j}^{1} \cdot \bar{\delta}_{1}\right)+\sum_{k=2}^{p-1}\left(\left(\bar{\delta}_{k}\right)\left(\mu_{i j}^{m} \cdot \bar{\delta}_{k}\right)\right)- \\
& -\sum_{k=2}^{p-1}\left(\left(\frac{\tilde{\delta}_{k}}{\bar{\delta}_{k}}\right)\left(\sum_{m=1}^{k-1} \mu_{i j}^{m} \cdot \bar{\delta}_{m}\right)\right)-\frac{\tilde{\delta}_{p}}{\bar{\delta}_{p}}\left(\sum_{m=1}^{p-1} \mu_{i j}^{m} \cdot \bar{\delta}_{m}\right)+\frac{\tilde{\delta}_{p}}{\bar{\delta}_{p}}\left(\sum_{m=1}^{p} \mu_{i j}^{m} \cdot \bar{\delta}_{m}\right)= \\
& =a_{i j}^{0}+\mu_{i j}^{1} \cdot \tilde{\delta}_{1}+\sum_{m=2}^{p-1}\left(\mu_{i j}^{m} \cdot \vec{\delta}_{m}\right)+\mu_{i j}^{p} \cdot \vec{\delta}_{p}= \\
& =a_{i j}^{0}+\sum_{m=1}^{p} \mu_{i j}^{m} \cdot \tilde{\delta}_{m}= \\
& =a_{i j}^{0}+\sum_{m=1}^{p} \mu_{i j}^{m} \cdot\left(\delta_{m}-\delta_{m}^{\min }\right)=a_{i j}^{0}+\sum_{m=1}^{p} \mu_{i j}^{m} \cdot \delta_{m}-\sum_{m=1}^{p} \mu_{i j}^{m} \cdot \delta_{m}^{\min } \stackrel{(8)}{=} \\
& =\sum_{m=1}^{p} \mu_{i j}^{m} \cdot \delta_{m}^{\min }+f_{i j}+\sum_{m=1}^{p} \mu_{i j}^{m} \cdot\left(\delta_{m}-\delta_{m}^{\min }\right)= \\
& =\sum_{m=1}^{p} \mu_{i j}^{m} \cdot \delta_{m}+f_{i j} \stackrel{(2)}{=} \\
& \stackrel{(2)}{=} \Delta f_{i j}+f_{i j} \stackrel{(9)}{=} a_{i j}
\end{aligned}
$$

This confirms that $\sum_{k=0}^{2^{p}-1} \lambda_{k} \cdot a_{i j}^{k}=a_{i j}$ or $A=\sum_{k=0}^{2^{p}-1} \lambda_{k} \cdot A^{k}$.

The lemma is proved.

Lemma 2. If all matrices $A^{k} \quad \forall k \in \overline{\left\{0,2^{p}-1\right\}}$ satisfy the following equations

$$
Q^{k}-K B R^{-1} B^{T} K^{T}+K A^{k}+\left(A^{k}\right)^{T} K^{T}=0
$$

where

$$
Q^{k}=K B R^{-1} B^{T} K^{T}-K A^{k}-\left(A^{k}\right)^{T} K^{T}
$$

symmetric non-negative definite matrices and $R \in R^{m \times m}$, $K \in R^{n \times n}$ - symmetric positive definite matrices then each matrix

$$
A=\sum_{k=0}^{2^{p}-1} \lambda_{k} \cdot A^{k}
$$

satisfies the following equation

$$
Q^{*}-K B R^{-1} B^{T} K^{T}+K A+A^{T} K^{T}=0
$$

here

$$
Q^{*}=\sum_{k=0}^{2^{p}-1} \lambda_{k} \cdot Q^{k}
$$

Proof

Conditions (18), (19) must be satisfied. We will show it below.

$$
\begin{gathered}
\sum_{k-0}^{2^{p}-1} \lambda_{k} \cdot\left(Q^{k}-K B(R)^{-1}(B)^{T}(K)^{T}+K A^{k}+\left(A^{k}\right)^{T}(K)^{T}\right)=0 \\
\sum_{k=0}^{2^{p}-1}\left(\lambda_{k} Q^{k}-\lambda_{k} K B(R)^{-1}(B)^{T}(K)^{T}+\lambda_{k} K A^{k}+\lambda_{k}\left(A^{k}\right)^{T}(K)^{T}\right)=0 \\
\sum_{k=0}^{2^{p}-1}\left(\lambda_{k} Q^{k}\right)-\left(K B(R)^{-1}(B)^{T}(K)^{T}\right) \sum_{k-0}^{2^{p}-1} \lambda_{k}+ \\
+K \sum_{k=0}^{2^{p}-1}\left(\lambda_{k} A^{k}\right)+\left(\sum_{k-0}^{2^{p}-1}\left(\lambda_{k} A^{k}\right)\right)^{T}(K)^{T}=0
\end{gathered}
$$

Such that from (10), (12), (19) and (22) we see that (18)

$$
Q^{*}-K B R^{-1} B^{T} K^{T}+K A+A^{T} K^{T}=0
$$

From (11), (19) we see that $Q^{*}$ is a symmetric nonnegative definite matrix.

The lemma is proved.

Comment. If $\Delta F \neq[0]$ then the optimal control (5) is such that the next cost function is minimized

$$
J^{*}=\int_{0}^{\infty}\left(x^{T} Q^{*} x+u^{T} R u\right) d t=x_{0}^{T} K x_{0}
$$

We also note that the cost function (23) has a matrix $Q^{*}$ which is dependent on $A=F+\Delta F$. Therefore, the cost function (23) has a variable structure.

\subsection{Estimation of the cost function}

Theorem 1. Suppose (1) is LIDS and the optimal control $\mathrm{u}$ can be represented as (5). Then

$$
J=\int_{0}^{\infty}\left(x^{T} Q x+u^{T} R u\right) d t \leq L\left(x_{0}^{T} K x_{0}\right)
$$

Where inequality (24) is an estimate of the cost function (6). Where $L=\max _{i, k}\left(\lambda_{i}^{k}\right)$ maximum of solutions to the equation: 


$$
\begin{aligned}
& \operatorname{det}\left(\left(Q+K B R^{-1} B^{T} K^{T}\right)-\lambda_{i}^{k}\left(Q^{k}+K B R^{-1} B^{T} K^{T}\right)\right)=0 \\
& i \in \overline{\{1, n\}}, k \in \overline{\left\{0,2^{p}-1\right\}}
\end{aligned}
$$

where

$$
P=\left(Q+K B R^{-1} B^{T} K^{T}\right) ; P^{k}=\left(Q^{k}+K B R^{-1} B^{T} K^{T}\right)
$$

Symmetric positive definite matrices of corresponding dimensions.

\section{Proof}

It is known that each of equations (25) has n real solutions.

We accept without proof the following fact. If

$$
\begin{gathered}
P=\left(Q+K B R^{-1} B^{T} K^{T}\right) ; P^{k}=\left(Q^{k}+K B R^{-1} B^{T} K^{T}\right), \\
\forall k \in \overline{\left\{0,2^{p}-1\right\}}
\end{gathered}
$$

are symmetric positive definite matrices, then

$$
\begin{gathered}
\left(\left(Q+K B R^{-1} B^{T} K^{T}\right)-L\left(Q^{k}+K B R^{-1} B^{T} K^{T}\right)\right), \\
\forall k \in \overline{\left\{0,2^{p}-1\right\}}
\end{gathered}
$$

There are symmetric non-positively defined matrices. Then

$$
\begin{aligned}
& x^{T}\left(\left(Q+K B R^{-1} B^{T} K^{T}\right)\right.\left.-L\left(Q^{k}+K B R^{-1} B^{T} K^{T}\right)\right) x \leq 0, \\
& \forall k \in \overline{\left\{0,2^{p}-1\right\}} \\
& x^{T}\left(Q+K B R^{-1} B^{T} K^{T}\right) x-x^{T} L\left(Q^{k}+K B R^{-1} B^{T} K^{T}\right) x \leq 0, \\
& \forall k \in \overline{\left\{0,2^{p}-1\right\}} \\
& x^{T}\left(Q+K B R^{-1} B^{T} K^{T}\right) x \leq x^{T} L\left(Q^{k}+K B R^{-1} B^{T} K^{T}\right) x, \\
& \forall k \in \overline{\left\{0,2^{p}-1\right\}} \\
& \sum_{k=0}^{2^{p-1}} \lambda_{k} x^{T}\left(Q+K B R^{-1} B^{T} K^{T}\right) x \leq \sum_{k=0}^{2^{p}-1} \lambda_{k} x^{T} L\left(Q^{k}+K B R^{-1} B^{T} K^{T}\right) x
\end{aligned}
$$

Where coefficients $\lambda_{k}$ satisfy (10), (11), (12). Moreover

$x^{T}\left(\sum_{k=0}^{2 p-1} \lambda_{k} Q+\sum_{k=0}^{2 p-1} \lambda_{k} K B R^{-1} B^{T} K^{T}\right) x \leq L x^{T}\left(\sum_{k=0}^{2 p^{p-1}} \lambda_{k} Q_{k}+\sum_{k=0}^{2 p^{p-1}} \lambda_{k} K B R^{-1} B^{T} K^{T}\right) x$

Such that, from (10), (11), (12), (19) we see that

$$
x^{T}\left(Q+K B R^{-1} B^{T} K^{T}\right) x \leq L x^{T}\left(Q^{*}+K B R^{-1} B^{T} K^{T}\right) x
$$

Such that, from (26) we see that

$$
\int_{0}^{\infty} x^{T}\left(Q+K B R^{-1} B^{T} K^{T}\right) x d t \leq L \int_{0}^{\infty} x^{T}\left(Q^{*}+K B R^{-1} B^{T} K^{T}\right) x d t
$$

From (27), (5) it follows that

$$
\int_{0}^{\infty}\left(x^{T} Q x+u^{T} R u\right) d t \leq L \int_{0}^{\infty}\left(x^{T} Q^{*} x+u^{T} R u\right) d t
$$

Finally, it follows from (28) and (20) that

$$
J=\int_{0}^{\infty}\left(x^{T} Q x+u^{T} R u\right) d t \leq L \int_{0}^{\infty}\left(x^{T} Q^{*} x+u^{T} R u\right) d t=L\left(x_{0}^{T} K x_{0}\right)
$$

This confirms that

$$
J=\int_{0}^{\infty}\left(x^{T} Q x+u^{T} R u\right) d t \leq L\left(x_{0}^{T} K x_{0}\right)
$$

The theorem is proved.

\subsection{Example}

Equation represents a production model

$$
T \dot{y}(t)+y(t)=\left(K_{o}+d K\right) u(t)
$$

Where $d K$, unknown constant and such that

$$
d K_{\min } \leq d K \leq d K_{\max }
$$

where $d K_{\min }, d K_{\max }, T, K_{o}$, these are known constants.

Suppose

$$
u(t)=-\left(K_{p} y(t)+I_{p} \int_{0}^{t} y(\tau) d \tau\right)
$$

This is a family of fixed structure controllers, that is called the controller family PI. Let $\dot{x}_{1}=x_{2} ; y=x_{2}$, then equation (30) can be expressed as (1), where $\mathrm{x} \in R^{2}$ state vector, $\mathrm{u} \in R^{1}$ control signal and $F=\left(f_{i j}\right) \in R^{2 \times 2}$, $\Delta F=\left(\Delta f_{i j}\right) \in R^{2 \times 2}$ and $B=\left(b_{i j}\right) \in R^{2 \times 1}$.

Matrix elements $F, \Delta F, B$ can be expressed as

$$
F=\left(\begin{array}{cc}
0 & 1 \\
0 & -\frac{1}{T}
\end{array}\right) \Delta F=\left(\begin{array}{cc}
\Delta f_{11} & \Delta f_{12} \\
\Delta f_{21} & \Delta f_{22}
\end{array}\right) B=\left(\begin{array}{c}
0 \\
K_{o} / T
\end{array}\right)
$$

$\Delta f_{11}=0 ; \Delta f_{12}=0 ; \Delta f_{21}=\mu_{21}^{1} \cdot \delta_{1} ; \Delta f_{22}=\mu_{22}^{1} \cdot \delta_{1} ; \delta_{1}=d K ;$

$$
\begin{gathered}
\mu_{21}^{1}=-\frac{I_{p}}{T} ; \mu_{22}^{1}=-\frac{K_{p}}{T} ; \\
d K_{\min }=\delta_{1}^{\min } \leq \delta_{1} \leq \delta_{1}^{\max }=d K_{\text {max }}
\end{gathered}
$$

If $\dot{x}_{1}=x_{2} ; y=x_{2}$, then equation (31)

$$
u(t)=-I_{p} \cdot x_{1}(t)-K_{p} \cdot x_{2}(t)
$$

Assume (32) is equivalent to (5):

$$
\left(\begin{array}{ll}
I_{p} & K_{p}
\end{array}\right)=R^{-1} B^{T} K^{T}
$$

Let 


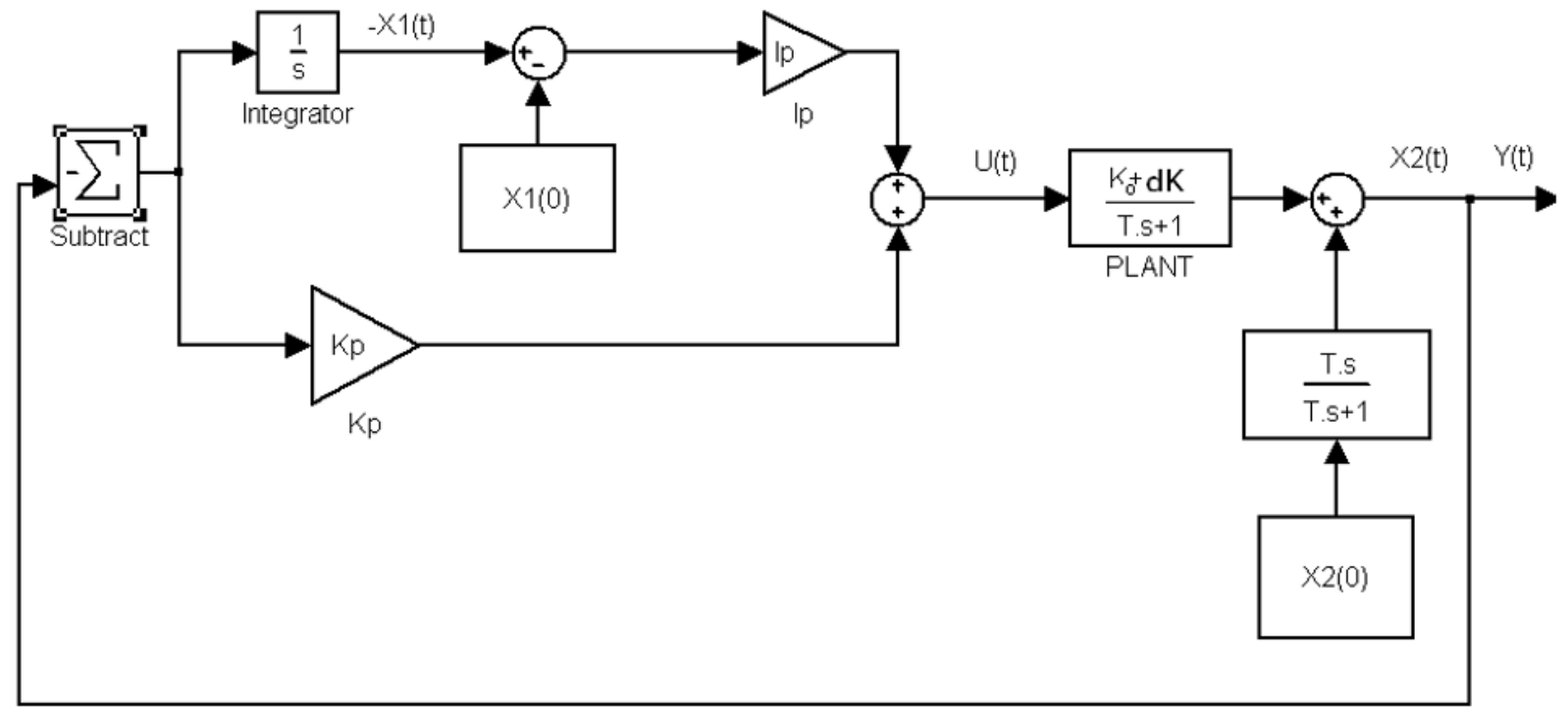

Fig. 1. Management system.

$$
Q=\left(\begin{array}{cc}
1 & 0 \\
0 & 1
\end{array}\right) ; R=(1) ; K=\left(\begin{array}{ll}
\mathrm{k}_{11} & \mathrm{k}_{12} \\
\mathrm{k}_{12} & \mathrm{k}_{22}
\end{array}\right)
$$

then the cost function

$$
J=\int_{0}^{\infty}\left(x_{1}^{2}(t)+x_{2}^{2}(t)+u^{2}(t)\right) d t=x_{0}^{T}\left(\begin{array}{ll}
\mathrm{k}_{11} & \mathrm{k}_{12} \\
\mathrm{k}_{12} & \mathrm{k}_{22}
\end{array}\right) x_{0}
$$

Where $x_{0}=\left(x_{1}(0) \quad x_{2}(0)\right)^{T}$, initial value of the state space $x(t) \in R^{2}$.

In this case, it is necessary to obtain an estimate of the cost function (33).

Figure 1 shows the influence of the initial conditions $x_{1}(0), x_{2}(0)$ on the control system.

According to (7), (8)

$$
\begin{aligned}
& \left.A^{0}=\left(\begin{array}{cc}
0 & 1 \\
-\frac{I_{p}}{T} d K_{\min } & \left(-\frac{1}{T}-\frac{K_{p}}{T} d K_{\min }\right.
\end{array}\right)\right) ;
\end{aligned}
$$

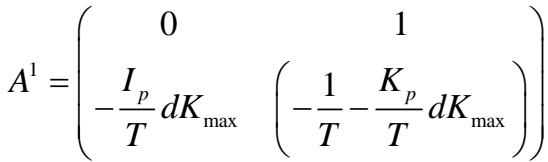

Let

$$
Q^{0}=K B R^{-1} B^{T} K^{T}-K A^{0}-\left(A^{0}\right)^{T} K^{T}
$$

and

$$
Q^{1}=K B R^{-1} B^{T} K^{T}-K A^{1}-\left(A^{1}\right)^{T} K^{T}
$$

where

$$
\begin{gathered}
P=\left(Q+K B R^{-1} B^{T} K^{T}\right) ; P^{0}=\left(Q^{0}+K B R^{-1} B^{T} K^{T}\right) ; \\
P^{1}=\left(Q^{1}+K B R^{-1} B^{T} K^{T}\right)
\end{gathered}
$$

symmetric positive definite matrices of corresponding dimensions.

Let $L=\max \left(\lambda_{i}\right)$, maximum between solutions of equations

$$
\begin{aligned}
& \operatorname{det}\left(\left(Q+K B R^{-1} B^{T} K^{T}\right)-\lambda\left(Q^{0}+K B R^{-1} B^{T} K^{T}\right)\right)=0 \\
& \operatorname{det}\left(\left(Q+K B R^{-1} B^{T} K^{T}\right)-\lambda\left(Q^{1}+K B R^{-1} B^{T} K^{T}\right)\right)=0
\end{aligned}
$$

We get an estimate of the cost function

$$
J=\int_{0}^{\infty}\left(x_{1}^{2}(t)+x_{2}^{2}(t)+u^{2}(t)\right) d t \leq L \cdot\left(x_{1}(0) \quad x_{2}(0)\right)\left(\begin{array}{ll}
\mathrm{k}_{11} & \mathrm{k}_{12} \\
\mathrm{k}_{12} & \mathrm{k}_{22}
\end{array}\right)\left(\begin{array}{l}
x_{1}(0) \\
x_{2}(0)
\end{array}\right)
$$

Note: According to figure $1-x_{1}(0)$ - initial value of the integrator PI-regulator and $x_{2}(0)$ - initial state of the controlled quantity.

\section{Results}

The main result of this work can be considered the upper bound (24) of the integral cost function (23).

\section{Conclusions}

In this paper, a technique is shown on the basis of which it is possible to synthesize regulators for various electric power systems.

From the set of these regulators, you can choose one in which the value of the evaluation of the form (24) will have the smallest value.

Using the results of this work, we can obtain controllers for electric power systems with interval 
characteristics and obtain the highest quality indicators of transients.

\section{References}

1. D. Tonn, S. Goremykin, N. Sitnikov, A. Mukonin, A. Pisarevsky, Improving the experimental technique of asynchronous single-phase motors equivalent circuits research (1258 AISC, p. 24-34, 2021).

2. Y. Frolov, V. Shelyakin, N. Sitnikov, S. Goremykin, D. Tonn, Modeling an induction motor based on the equations of a generalized electric machine, taking into account the saturation of the magnetic circuit (E3S Web of Conferences 178, 01011, 2020).

3. Yu. Pisarevsky, A. Pisarevsky, V. Fursov, S. Goremykin, N. Sitnikov, Prospective electric motors for high speed medical instrument (IOP Conference Series: Materials Science and Engineering 643(1), 012039, 2019).

4. V. Volkov, V. Sergeev, V. Shelyakin, N. Sitnikov, S. Goremykin, D. Mamontov, Innovative local heat supply stations for industrial buildings (IOP Conference Series: Materials Science and Engineering 643(1), 012097, 2019).

5. V. Akimov, A. Polukazakov, N. Sitnikov, Selecting Criteria for Optimizing Parameters of ADC for Digital Signal Processing (Proceedings - 2019 International Russian Automation Conference, RusAutoCon 2019 8867651). 